\title{
Muscidae e Calliphoridae (Diptera, Brachycera) associados à decomposição cadavérica no noroeste do Paraná
}

\author{
Ana Caroline Oliveira Pereira, Alex Sandro Barros de Souza ${ }^{\circledR}$ \& Patricia Pereira Gomes
}

Instituto Federal de Educação, Ciência e Tecnologia do Paraná - IFPR, Umuarama, PR, Brasil.

\author{
Entomology Beginners, vol. 2: e020 (2021)
}

\begin{abstract}
Resumo. O presente trabalho teve como objetivo listar quais são os dípteros necrófagos na região noroeste do Paraná. As informações fazem parte de um projeto, aprovado no comitê de ética em pesquisa de animais, em desenvolvimento no IFPR Campus Umuarama. Durante o estudo, um cadáver de suíno com o peso aproximado de $10 \mathrm{~kg}$ foi utilizado como substrato, o qual foi colocado em uma gaiola com uma tela, para impedir a entrada de vertebrados necrófagos. A coleta dos insetos foi realizada por meio de uma armadilha modificada. A gaiola de metal que continha o substrato estava fixada no terreno do IFPR Campus Umuarama. Em relação aos insetos foram coletadas cinco espécies de Muscidae (Sarcopromusca pruna; Musca domestica; Hydrotaea aenescens; Sythesiomyia nudiseta e Stomoxys calcitrans) e seis espécies de Calliphoridae (Cochliomyia macellaria; Chrysomya albiceps; Chrysomya megacephala; Lucilia eximia; Lucilia cuprina e Hemilucilia segmentaria). Os espécimes de Stomoxys calcitrans podem ter sido coletados devido à presença de gado de corte próximo ao local. O processo de decomposição se completou em seis dias, o que pode ser devido aos altos índices de temperatura e baixos índices de umidade observados no local. Nesse período foram observados quatro estágios: fresco; enfisematoso; coliquativo e esqueletização. Estes dados são importantes para a construção de um banco de dados de espécies de importância forense.
\end{abstract}

Palavras chave: Chrysomya; Decomposição; Entomologia Forense; Varejeiras.

\section{Muscidae and Calliphoridae (Diptera, Brachycera) associated with cadaveric decomposition in northwestern Paraná state, Brazil}

Abstract. This work aimed to list which are the scavenger dipterans in the Paraná state northwestern region. The information is part of a project, approved by the animal research ethics committee, under development at the IFPR Campus Umuarama, Brazil. During the study, a pig cadaver weighing $10 \mathrm{~kg}$ was used as a substrate, which was placed in a cage with a screen to prevent entry of scavenger vertebrates. Insects were collected using a modified trap. The metal cage containing the substrate was fixed on the IFPR Campus Umuarama grounds. Regarding insects, five Muscidae species were collected: (Sarcopromusca pruna; Musca domestica; Hydrotaea aenescens; Sythesiomyia nudiseta and Stomoxys calcitrans) and six Calliphoridae species (Cochliomyia macellaria; Chrysomya albiceps; Chrysomya megacephala; Lucilia eximia; Lucilia cuprina and Hemilucilia segmentaria). Specimens of Stomoxys calcitrans may have been collected due to the presence of beef cattle close to the site. The decomposition process was completed in six days, which may be due to the high temperature and low humidity levels observed at the site. During this period, four stages were observed: fresh; emphysematous; colliquative and skeletonization. These data are important for building a database of forensic importance species.

Keywords: Chrysomya; Decomposition; Forensic Entomology; Blowflies.

Insetos e outros artrópodes têm sido amplamente estudados pela Entomologia Forense (EF) para esclarecer questões criminais, tais como: quem é o cadáver?, quais as circunstâncias que levaram à morte?, o local onde ocorreu a morte e, principalmente, para definir o intervalo pós-morte (IPM) (OLIVEIRA-COSTA, 2013). O conhecimento sobre a biologia, taxonomia e ecologia dos insetos necrófagos é essencial para a $E F$, pois estes são os primeiros organismos a localizar o cadáver, assim que o processo de decomposição tem início, passando a utilizar rapidamente a matéria orgânica morta para concluir seu desenvolvimento (OLIVEIRA-COSTA, 2013).

Dentre as diversas espécies de insetos necrófagos, aquelas pertencentes à ordem Diptera, em especial às famílias
Calliphoridae e Muscidae, fornecem dados sólidos para a estimativa de tempo (IPM), pois geralmente são os primeiros insetos a serem atraídos pelo corpo após a morte, fazendo a colonização do substrato (CARVALHO e LINHARES, 2001). A família Calliphoridae possui mais de 1.500 espécies, reconhecidas popularmente como "moscas-varejeiras", possuem tamanho médio, apresentam as regiões do tórax e abdome de cor metálica e são encontradas em todas as regiões biogeográficas (BROWN et al., 2009). Já a família Muscidae, com mais de 9.000 espécies ao redor do mundo, possui espécies de tamanhos variados, contendo aparelho bucal com duas formas (sugador ou lambedor), além de apresentarem caliptras (CARVALHO e MELLO-PATIU, 2008). Este estudo teve, portanto,

\section{Editado por:}

William Costa Rodrigues

\section{Histórico Editoral:}

Recebido em: 23.07.2021

Aceito em: 13.09.2021

Publicado em: 05.10 .2021
Autor Correspondente:

Alex Sandro Barros de Souza

alex.desouza@ifpr.edu.br

Agência(s) de Fomentos:

Instituto Federal de Educação, Ciência e Tecnologia do Paraná - IFPR 
o objetivo de fornecer uma lista de espécies de Muscidae e Calliphoridae associados à decomposição animal no município de Umuarama, noroeste do Paraná, Brasil.

Este estudo foi aprovado pela comissão de ética no uso de animais (CEUA) do Instituto Federal do Paraná, processo número 009569/2019-12. O experimento de campo foi executado em um fragmento florestal $\left(23^{\circ} 48^{\prime} 21,5^{\prime \prime} \mathrm{S}\right.$ e $\left.53^{\circ} 19^{\prime} 26,8^{\prime \prime} \mathrm{O}\right)$ localizado no Campus do Instituto Federal de Educação, Ciência e Tecnologia do Paraná - IFPR, Campus Umuarama, PR. O experimento foi executado entre quatro a dez de setembro de 2019. Como substrato atrativo foi utilizado um cadáver suíno (Sus scrofa L.) com peso aproximado de $10 \mathrm{~kg}$. A carcaça animal foi doada pelo Hospital Veterinário da UNIPAR. O cadáver foi colocado em contato com o solo, dentro de uma gaiola de dimensões de $160 \times 40 \times 68 \mathrm{~cm}$ (Figuras 1 A a D), revestida com tela com espaçamento de $3 \times 3 \mathrm{~cm}$, para permitir a entrada de insetos e ao mesmo tempo impedir o acesso de vertebrados de grande porte existentes na área de estudo. A identificação dos adultos seguiu as chaves Carvalho e Mello-Patiu (2008) e Kosmann et al. (2013). A coleta dos insetos foi realizada por meio de uma armadilha modificada (Figura 1E), após a coleta os espécimes adultos foram sacrificados por resfriamento (-1 ${ }^{\circ} \mathrm{C}$ durante 15 minutos) e transferidos para frascos com álcool 70\%, e posteriormente depositados na Coleção DidáticoCientífica do IFPR, Campus Umuarama, PR. A nomenclatura dos estágios de decomposição segue a proposta de Bornemissza (1957). Os dados climatológicos do período foram fornecidos pela Estação Climatológica do SIMEPAR (Sistema Meteorológico do Paraná). As médias de temperatura, umidade relativa do ar e precipitação para o período de estudo foram: $28,2{ }^{\circ} \mathrm{C}, 61 \%$ e $0,3 \mathrm{~mm}$.
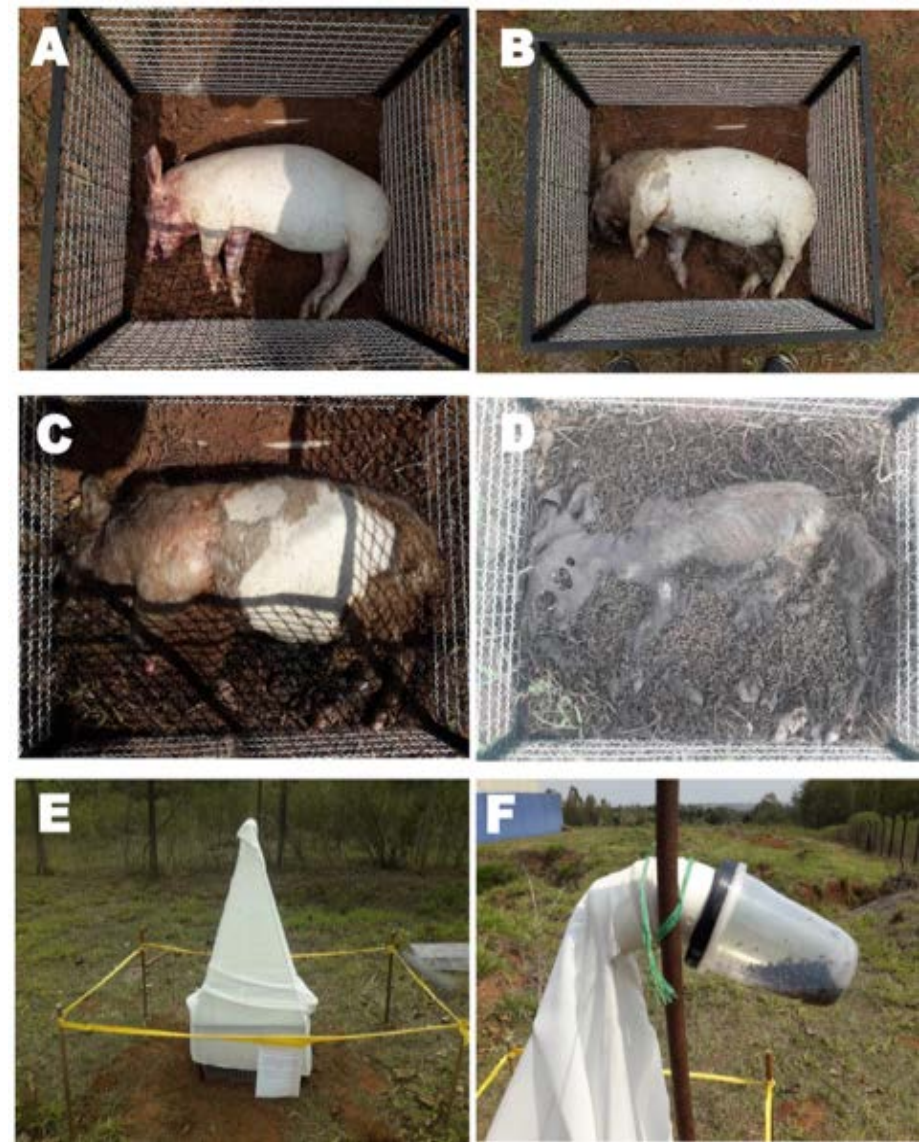

Figura 1. A. Fresco; B. Enfisematoso; C. Coliquativo; D. Esqueletização; E. Gaiola de coleta com armadilha suspensa; F. Copo coletor com Diptera. (Crédito das imagens: Alex Souza).

Foram observados quatro estágios de decomposição (Figuras 1 A a D): fresco; enfisematoso; coliquativo e esqueletização, sendo que este último estágio foi acompanhado de uma mumificação da pele que não estava em contato com o solo. A decomposição durou cerca de seis dias, ocorrendo rapidamente quando comparada a estudos com animais de pesos similares (CARVALHO e LINHARES, 2001), podendo ser devido aos altos índices de temperatura e baixos índices de umidade, apresentados na região durante o período do experimento (temperaturas atípicas para o inverno), o que pode ter acelerado o metabolismo dos imaturos. Quanto à colonização dos imaturos, foi observada uma grande incidência de indivíduos imaturos de Chrysomya albiceps, as quais praticamente compunham a totalidade de imaturos encontrados na carcaça, as larvas de terceiro estágio de $C$. albiceps são de fácil identificação visual, devido aos seus longos tubérculos dorsais (QUEIROZ et al., 1997). Além disso, estas larvas possuem um curto tempo de desenvolvimento quando comparadas às espécies nativas (BARROS-SOUZA et al., 2012), possuindo também um alto poder de predação (GALINDO et al., 2016; SPINDOLA et al., 2017). O gênero Chrysomya foi introduzido no Brasil na década de 1970 (GUIMARÃES et al., 1978) e sendo espécies invasoras altamente sinantrópicas, muito bem adaptadas ao ambiente humano, rapidamente distribuíram-se pelo território brasileiro (FERRAZ et al., 2010).

As espécies registradas neste trabalho também foram observadas em estudos prévios realizados em carcaças de suínos e cadáveres humanos em outras regiões do Brasil (CAVALCANTE et al., 2015; GAEDKE e MOUGA, 2017; FARIA et al., 2018). Hydrotaea aenescens e Musca domestica foram os muscídeos mais abundantes na carcaça (Tabela 1). Hydrotaea aenescens utiliza vários substratos de criação e em alguns casos suas larvas podem ser predadoras facultativas (D'ALMEIDA et al., 1999). Espécies de Hydrotaea sp. e M. domestica têm sido encontradas em carcaças de animais e cadáveres humanos em ambientes urbanos em vários países, incluindo o Brasil (CARVALHO e LINHARES, 2001; CARVALHO et al., 2004; KIMBERLY et al., 2005). Quanto a Stomoxys calcitrans, embora tenha tido presença de gado de corte próximo ao local onde foi realizado o experimento, esta espécie já foi coletada em outros estudos de EF no Brasil (ROSA et al., 2009, BARBOSA e VASCONCELOS, 2018). Apesar de Calliphoridae ser um grupo relativamente bem estudado, a fauna do Paraná, especificamente, ainda é pouco conhecida. O primeiro registro foi feito em um estudo de sinantropia realizado por Ferreira (1978) que listou oito espécies em Curitiba. Posteriormente, estudos com foco em EF, realizados por Moura et al. (1997) e Caleffe et al. (2015), registraram, cada um, três espécies de Calliphoridae. Sendo assim, o presente estudo torna-se importante por apresentar uma diversidade de Calliphoridae superior aos dois estudos realizados anteriormente no Estado.

Tabela 1. Distribuição de indivíduos de cada espécie de Calliphoridae e Muscidae coletados.

\begin{tabular}{lc}
\hline \multicolumn{1}{c}{ Táxon } & Número \\
\hline Calliphoridae & \\
\hline Chrysomya albiceps (Wiedemann, 1819) & 428 \\
\hline Chrysomya megacephala (Fabricius, 1794) & 231 \\
\hline Cochliomyia macellaria (Fabricius, 1775) & 02 \\
\hline Hemilucilia segmentaria (Fabricius, 1805) & 68 \\
\hline Lucilia cuprina (Wiedemann, 1830) & 12 \\
\hline Lucilia eximia (Wiedemann, 1819) & 74 \\
\hline Muscidae & \\
\hline Hydrotaea aenescens (Wiedemann, 1830) & 93 \\
\hline Musca domestica (Linnaeus, 1758) & 102 \\
\hline Sarcopromusca pruna (Shannon e Del Ponte, 1926) & 07 \\
\hline Stomoxys calcitrans (Linnaeus, 1758) & 28 \\
\hline Sythesiomyia nudiseta (Wulp, 1883) & 05 \\
\hline
\end{tabular}


A espécie mais abundante foi C. albiceps, seguida de Chrysomya megacephala, as quais são consideradas sinantrópicas em várias regiões no Brasil (VIANA et al., 1998) e comumente coletadas em estudo de EF no país (CAVALCANTE et al., 2015; GAEDKE e MOUGA, 2017; FARIA et al., 2018). A espécie Cochliomyia macellaria foi a menos coletada do estudo, apenas dois indivíduos (Tabela 1). Esta espécie é relativamente comum no território brasileiro, todavia é muito sensível à ação de $C$. albiceps (FARIA et al., 1999; GALINDO et al., 2016), espécie mais abundante no presente estudo. Um trabalho prévio verificou, inclusive, que $C$. albiceps tende a preferir predar larvas de $C$. macellaria em relação a outras espécies estudadas (FARIA et al., 1999). Quando comparado a trabalhos de EF realizados na região sul do Brasil (MOURA et al., 1997; SOUZA et al., 2008; GAEDKE e MOUGA, 2017), o resultado obtido neste estudo é expressivo, visto que muitas das espécies de Muscidae e Calliphoridae de interesse forense que ocorrem no sul do Brasil, foram coletadas. Os resultados também reforçam a necessidade de estudos localizados de EF, para poder visualizar com precisão a decomposição cadavérica e a fauna associada à decomposição animal, visto que cada região possui características únicas e isto influencia em todo o processo de decomposição e os insetos possuem seu metabolismo influenciado por fatores abióticos como temperatura e umidade (BROWN et al., 2009), podendo acelerar ou retardar seu metabolismo, afetando assim a velocidade do processo de decomposição.

\section{AGRADECIMENTOS}

Os autores agradecem ao Instituto Federal de Educação, Ciência e Tecnologia do Paraná - IFPR pela concessão da bolsa PIBIC edital 05/2019.

\section{REFERÊNCIAS}

BARBOSA, T. M.; VASCONCELOS, S. D. Muscidae (Diptera) of medico-legal importance associated with ephemeral organic substrates in seasonally dry tropical forests. Papéis Avulsos de Zoologia, v. 58, p. e20185826, 2018. DOI: https://doi.org/10.11606/1807-0205/2018.58.26

BARROS-SOUZA, A. S.; KEPPLER, R. L. F.; AGRA, D. B. Development period of forensic Importance Calliphoridae (Diptera: Brachycera) in urban area under natural conditions in Manaus, Amazonas, Brazil. EntomoBrasilis, v. 5, p. 88-92, 2012. DOI: https://doi.org/10.12741/ebrasilis.v5i2.201

BORNEMISSZA, G. F. An analysis of arthropod succession in carrion and the effect of its decomposition on the soil fauna. Australian Journal of Zoology, v. 5, p. 1-12, 1957. DOI: https://doi.org/10.1071/ZO9570001

BROWN, B. V.; BORKENT, A.; CUMMING, J. M.; WOOD, D. M.; WOODLEY, N. E.; ZUMBADO, M. A. Manual of Central America Diptera. Volume 1. Ottawa: NRC Research Press, 2009. 714p.

CALEFFE, R.; OLIVEIRA, S.; NANYA, S.; CONTE, H. Calliphoridae (Diptera) de interesse forense com ocorrência em Maringá-PRBrasil. Revista Uningá, v. 43, n. 1, p. 10-15, 2015.

CARVALHO, L. M. L; LINHARES, A. X. Seasonality of insect succession and pig carcass decomposition in a natural forest area in Southeastern Brazil. Journal of Forensic Sciences, v. 46, n. 3, p. 604-608, 2001.

CARVALHO, L. M. L.; THYSSEN, P. J.; GOFF M. L.; LINHARES,
A. X. Observations on succession patterns of necrophagous insects on pig carcass in a urban area of Southeastern Brazil. Anil Aggrawal's Internet Journal of Forensic Medicine and Toxicology, v. 5, p. 40-44, 2004.

CARVALHO, C. J. B.; MELLO-PATIU, C. A. Key to the adults of most common forensic species of Diptera in South America. Revista Brasileira de Entomologia, v. 52, n. 3, p. 390-406, 2008. DOI: http://doi.org/10.1590/S0085-56262008000300012

CAVALCANTE, A. N. P.; DAL-BÓ, D.; CREÃO-DUARTE, A. J.; FARIAS, R. C. A. P. Espécies de Calliphoridae (Diptera) associadas a carcaças de Sus scrofa Linnaeus, 1758 em área de restinga na Paraíba, Brasil, e espécies de importância forense para a estimativa do Intervalo Pós-Morte (IPM). Entomotropica, v. 30, p. 150-159, 2015.

D'ALMEIDA,J.M.;BORGES, C.;GONÇALVES, C. A. Desenvolvimento pós-embrionário de Ophyra aenescens (Wiedemann, 1830) (Diptera: Muscidae) em diferentes dietas, sob condições de laboratório. Memórias do Instituto Oswaldo Cruz, v. 94, p. 123-126, 1999.

FARIA, L. D. B.; ORSI, L.; TRINCA, L. A.; GODOY, W. A. C. Larval predation by Chrysomya albiceps on Cochliomyia macellaria, Chrysomya megacephala and Chrysomya putoria. Entomologia Experimentalis et Applicata, v. 90, p. 149-155, 1999. DOI: https://doi.org/10.1046/j.1570-7458.1999.00433.x

FARIA, L. S.; PASETO, M. L.; COURI, M. S.; MELLOPATIU, C. A.; MENDES, J. Insects Associated with pig carrion in two environments of the Brazilian Savanna. Neotropical Entomology, v. 47, p. 181-198, 2018. DOI: https://doi.org/10.1007/s13744-017-0518-y

FERRAZ, A. C. P.; GADELHA, B. Q.; QUEIROZ, M. M. C.; MOYABORJA, G. E.; AGUIAR-COELHO, V. M. Effects of forest fragmentation on dipterofauna (Calliphoridae) at the Reserva Biológica do Tinguá, Nova Iguaçu, RJ. Brazilian Journal of Biology, v. 70, n. 1, p. 55-63, 2010. DOI: https://doi.org/10.1590/ S1519-69842010000100009

FERREIRA, M. J. M. Sinantropia de dípteros muscóides de Curitiba, Paraná. I. Calliphoridae. Revista Brasileira de Biologia, v. 38, n. 2, p. 445-454, 1978.

GAEDKE, A.; MOUGA, D. M. D. S. Diptera survey in human corpses in the north of the state of Santa Catarina, Brazil. Acta Biológica Catarinense, v. 4, p. 42-51, 2017. DOI: https://doi.org/10.21726/abc.v4i1.359

GALINDO, L. A.; MORAL, R. A.; MORETTI, T. C. Intraguild predation influences oviposition behavior of blow flies (Diptera: Calliphoridae). Parasitology Research, v. 115, p. 2097-2102, 2016. DOI: https://doi.org/10.1007/s00436-016-4954-0

GUIMARÃES, J. H.; PRADO, A. P.; LINHARES, A. X. Tree newly introduced blowfly species in southern Brazil (Diptera, (alliphoridae). Revista Brasileira de Entomologia, v. 22, p. 53-60, 1978. 
KIMBERLY, L. T.; FELL, R. D.; BREWSTER, C. C. Insect fauna visiting carrion in Southwest Virginia. Forensic Science International, v. 150, p. 73-80, 2005.

KOSMANN, C.; MELLO, R. P.; HARTERREITEN-SOUZA, E. S.; PUJOL-LUZ, J. R. A List of current valid blow fly names (Diptera: Calliphoridae) in the Americas South of Mexico with key to the Brazilian species. EntomoBrasilis, v. 6, n. 1, p. 74-85, 2013. DOI: https://doi.org/10.12741/ebrasilis.v6i1.266

MOURA, M. O.; CARVALHO, C. J. B.; MONTEIRO-FILHO, E. L. A. A Preliminary analysis of insects of medico-legal Importance in Curitiba, State of Paraná. Memórias do Instituto Oswaldo Cruz, v. 92, n. 2, p. 269-274, 1997. DOI: https://doi.org/10.1590/ s0074-02761997000200023

OLIVEIRA-COSTA, J. (Ed.). Insetos "peritos": A Entomologia Forense no Brasil. $1^{\text {a }}$ ed. Campinas: Editora Millennium, 2013. 488p.

QUEIROZ, M. M. C.; MELLO, R. P.; LIMA, M. M. Morphological aspects of the larval instars of Chrysomya albiceps (Diptera,
Calliphoridae) reared in the laboratory. Memórias do Instituto Oswaldo Cruz, v. 92, n. 2, p. 187-196, 1997. DOI: https://doi.org/10.1590/S0074-02761997000200010

ROSA, T. A.; BABATA, M. L. Y.; SOUZA, C. M. S.; MELLOPATIU, C. A.; MENDES, J. Dípteros de interesse forense em dois perfis de vegetação de cerrado em Uberlândia, MG. Neotropical Entomology, v. 38, n.6, p. 859-866, 2009. DOI: https://doi.org/10.1590/S1519-566X2009000600022

SOUZA, A. S. B.; KIRST, F. D.; KRUGER, R. F. Insects of forensic importance from Rio Grande do Sul state in southern Brazil. Revista Brasileira de Entomologia, v. 52, p. 641-646, 2008. DOI: https://doi.org/10.1590/S0085-56262008000400016.

SPINDOLA, A. F.; ZHENG, L.; TOMBERLIN, J. K.; THYSSEN, P. J. Attraction and oviposition of Lucilia eximia (Diptera: Calliphoridae) to resources colonized by the invasive competitor Chrysomya albiceps (Diptera: Calliphoridae). Journal of Medical Entomology, v. 54, n. 2, p. 321-328, 2017. DOI: https://doi.org/10.1093/jme/tjw170 